\title{
ON THE INTEGRABILITY OF ALMOST CONTACT STRUCTURE
}

\author{
Shigeo SASAki AND Chen-Jung Hsu' ${ }^{1)}$
}

(Received Feb. 5, 1962)

A $(2 n+1)$-dimensional differentiable manifold $M^{2 n+1}$ of class $C^{\omega}$ is said to have a $(\phi, \xi, \eta)$-structure $[1]^{2)}$ (or an almost contact structure) if there exist a tensor field $\phi_{j}^{i}$, a contravariant vector field $\xi^{i}$ and a covariant vector field $\eta_{j}$ of class $C^{\omega}$ over $M^{2 n+1}$ such that the following conditions are satisfied:

$$
\begin{aligned}
\xi^{i} \eta_{i} & =1, \\
\operatorname{rank}\left|\phi_{j}^{i}\right| & =2 n, \\
\phi_{j}^{i} \xi^{j} & =0, \\
\phi_{j}^{i} \eta_{i} & =0, \\
\phi_{j}^{i} \phi_{k}^{j} & =-\delta_{k}^{i}+\xi^{i} \eta_{k} .
\end{aligned}
$$

It is shown [2] that one can define by a natural way a $3-\pi$-structure whose fundamental tensor is written as

(0. 2) $\quad F_{j}^{i}=\frac{1}{2}\left\{-\delta_{j}^{i}+3 \xi^{i} \eta_{j}+i \omega_{1}\left(1-\omega_{1}\right) \phi_{j}^{i}\right\}=\frac{1}{2}\left(-\delta_{j}^{i}+3 \xi^{i} \eta_{j}-\sqrt{3} \phi_{j}^{i}\right)$

and three kinds of $2-\pi$-structure whose fundamental tensors are respectively written as

$$
\left\{\begin{array}{l}
F_{j}^{i}=2 \xi^{i} \eta_{j}-\delta_{j}^{i}, \\
F_{j}^{i}=\xi^{i} \eta_{j}-i \phi_{j}^{i}, \\
F_{j}^{i}=\xi^{i} \eta_{j}+i \phi_{j}^{i}
\end{array}\right.
$$

for any $(\phi, \xi, \eta)$-structure. Here a $3-\pi$-structure $(2-\pi$-structure) is by definition a structure defined by giving three (two) differentiable distributions which assign three (two) complementary subspaces of dimension $\geqq 1$ in the complexified tangent space $T_{x}^{C}$ at each point $x$ of the differentiable manifold $M^{2 n+1}$. The fundamental tensor of a $3-\pi$-structure (2- $\pi$-structure) is the one which has the three (two) subspaces stated above as the proper subspaces corresponding to the proper values, which are cubic (or quadratic) roots of unity, of the linear transformation induced by it at the tangent space of each point of $M^{2 n+1}$.

The torsion tensor (an analogue of Nijenhuis tensor) of the 3- $\pi$-structure

1) This research work of the second author is partly supported by a grant from The National Council on Science Development.

2) Number in bracket refers to the reference at the end of the paper. 
(0.2) is

$$
\begin{aligned}
t_{j k}^{i}=\frac{1}{4}\left\{-N_{j k}^{i}\right. & -3 \xi^{i}\left(\eta_{j}, k-\eta_{k},{ }_{j}\right)+5 \xi^{i}\left(N_{j} \eta_{k}-N_{k} \eta_{j}\right) \\
& \left.+\xi^{i} \phi_{j}^{\eta} \phi_{k}^{q}\left(\eta_{q, p}-\eta_{p}, q\right)-N_{p}^{i}\left(\phi_{j}^{p} \eta_{k}-\phi_{k}^{p} \eta_{j}\right)\right\},
\end{aligned}
$$

and those of $2-\pi$-structures $\left(\begin{array}{lll}0 & 3\end{array}\right)$ are respectively

$$
\left\{\begin{array}{l}
t_{j k}^{i}=-\xi^{i}\left(-N_{j} \eta_{k}+N_{k} \eta_{j}+\eta_{j, k}-\eta_{k}, j\right) \\
t_{j k}^{i}=-\frac{1}{4} P_{j k}^{i}-\frac{1}{4} i Q_{j k}^{i}, \\
t_{j}^{i}=-\frac{1}{4} P_{j k}^{i}+\frac{1}{4} i Q_{j k}^{i} .
\end{array}\right.
$$

In these formulas we have put

$$
\left\{\begin{array}{l}
P_{j k}^{i}=N_{j k}^{i}-t_{j k}^{i}-\xi^{i}\left(\eta_{j, k}-\eta_{k}, j\right)=N_{j k}^{i}-\xi^{i}\left(N_{j} \eta_{k}-N_{k} \eta_{j}\right), \\
Q_{j k}^{i}=-N_{j}^{i} \eta_{k}+N_{k}^{i} \eta_{j}+\xi^{i} N_{j k}
\end{array}\right.
$$

where $\eta_{j, k}=\frac{\partial \eta_{j}}{\partial x^{k}}$, and the tensors $N_{j k}^{i}, N_{j}^{i}, N_{j k}, N_{j}$ are defined as follows [3]:

$$
\left\{\begin{array}{l}
N_{j k}^{i}=\phi_{k}^{i}\left(\phi_{j, q}^{i}-\phi_{4, j}^{i}\right)-\phi_{j}^{p}\left(\phi_{i, p}^{i}-\phi_{1, k}^{i}\right)-\eta_{j} \xi_{, k}^{i}+\eta_{k} \xi_{, j}^{i}, \\
N_{j k}=\phi_{k}^{q}\left(\eta_{q, j}-\eta_{j, q}\right)-\phi_{j}^{p}\left(\eta_{p, k}-\eta_{k, p}\right), \\
N_{j}^{i}=\xi^{q}\left(\phi_{j, q}^{i}-\phi_{4, j}^{i}\right)-\phi_{j, q}^{i} \xi_{,}^{i}, \\
N_{j}=\xi^{p}\left(\eta_{j, p}-\eta_{p, j}\right) .
\end{array}\right.
$$

In this short note, we intend to study the characterization of the vanishing of $N_{j k}^{i}$ geometrically in $M^{2 n+1}$ which is an open question in [4]. Moreover, some related problems are also considered.

1. It is known [5] that the vanishing of torsion tensor $t_{j k}^{i}$ in (0.4) is a necessary and sufficient condition for the $3-\pi$-structure defined by $(0.2)$ to be integrable and the vanishing of respective tensor in (0.5) is the condition of respective $2-\pi$-structure in (0.3) to be integrable. By definition, a $3-\pi$-structure defined by three distributions $T_{1}, T_{2}, T_{3} ; \operatorname{dim} T_{i}=\nu_{i}, \nu_{1}+\nu_{2}+\nu_{3}=2 n+1$ is said to be integrable if at each point of $M^{2 n+1}$ there exist a neighborhood and $2 n+1$ complex valued functions $z^{i}$ of the local coordinates in the neighborhood such that $T_{1}$ is expressed by $d z^{l_{1}+i}=\cdots \cdots=d z^{l_{1}+l_{2}+l_{3}}=0, T_{2}$ by $d z^{1}=\ldots \ldots$ $=d z^{v_{1}}=d z^{v_{1}+l_{2}+1}=\cdots \cdots=d z^{v_{1}+l_{2}+l_{3}}=0$ and $T_{3}$ by $d z^{1}=\cdots \cdots=d z^{v_{1}}=d z^{v_{1}+1}=\ldots \ldots$ $=d z^{r_{1}+r_{2}}=0$. The integrability of $2-\pi$-structure is similarly defined.

As the $3-\pi$-structure defined by $(0.2)$ is the refinement of each of the $2-\pi$ structures in (0.3), it is evident from the definition of integrability of $\pi$-structure that the vanishing of $t_{j k}^{i}$ in (0.4) implies the vanishing of all of torsion tensors of (0.5) and tensors $P_{j k}^{i}$ and $Q_{j k}^{i}$ in (0.6). But this can also be shown by direct 
calculation from (0.4) by making use of some of the following identities obtained in [3] :

(1. 1)

$$
\begin{aligned}
& \phi_{h}^{i} N_{j k}^{h}+N_{j h}^{i} \phi_{k}^{h}+\xi^{i} N_{j k}-N_{j} \eta_{k}=0 \\
& N_{j n}^{i} \xi^{h}+\phi_{h}^{i} N_{j}^{h}-\xi^{i} N_{j}=0 \\
& \eta_{h} N_{j k}^{h}-N_{j h} \phi_{k}^{h}-N_{j} \eta_{k}=0 \\
& \phi_{h}^{i} N_{j}^{h}+N_{h}^{i} \phi_{j}^{h}-\xi^{i} N_{j}=0 \\
& N_{h i}^{i} \xi^{h}=0 \\
& \eta_{h} N_{j}^{h}-N_{j h} \xi^{h}=0 \\
& \eta_{h} N_{j}^{h}+N_{h} \phi_{j}^{h}=0 \\
& N_{h} \xi^{h}=0 \\
& N_{j h}^{i} \phi_{k}^{h}-N_{h k}^{i} \phi_{j}^{h}-N_{j}^{i} \eta_{k}-N_{k}^{i} \eta_{j}=0 \\
& N_{j h}^{i} \xi^{h}-N_{h}^{i} \phi_{j}^{h}=0 \\
& N_{j h} \phi_{k}^{h}-N_{h k} \phi_{j}^{h}+N_{j} \eta_{k}-N_{k} \eta_{j}=0 \\
& N_{j h} \xi^{h}+N_{h} \phi_{j}^{h}=0 \\
& N_{j}=N_{h k} \phi_{j}^{h} \xi^{k} \\
& N_{j}=\eta_{h} N_{l}^{h} \phi_{j}^{l}, \\
& N_{j}=N_{i h}^{i} \xi^{h} \eta_{i} \\
& N_{j k}=-\eta_{i} N_{j h}^{i} \phi_{k}^{h}+N_{j}^{i} \eta_{i} \eta_{k} \\
& N_{j}^{i}=\phi_{h}^{i} N_{j k}^{h} \xi^{k}+\xi^{i} N_{j k} \xi^{k}
\end{aligned}
$$

Now, we prepare some formulas to be used later. Contract $\xi^{k}$ with $t_{j i}^{\prime}$ in (0.4), then we have the following by virtue of $(0.7)_{4},(1.1)_{5}$ and $(0.1)_{3}$ :

$$
t_{j k}^{i} \xi^{k}=\frac{1}{4}\left(-N_{j k}^{i} \xi^{k}+2 \xi^{i} N_{j}-N_{p}^{i} \phi_{j}^{p}\right) \text {. }
$$

Substitute $N_{i h}^{i} \xi^{k}$ from $(1.1)_{2}$, we have

$$
t_{j k}^{i} \xi^{k}=\frac{1}{4}\left(\phi_{p}^{i} N_{j}^{p}-N_{p}^{i} \phi_{j}^{p}+\xi^{i} N_{j}\right) .
$$

Finally, substitute from $(1.1)_{4}$ we have

$$
\begin{aligned}
t_{j k}^{i} \xi^{k} & =\frac{1}{2} \phi_{p}^{i} N_{j}^{p}, \quad \text { or } \\
t_{j k}^{i} \xi^{k} & =\frac{1}{2}\left(\xi^{i} N_{j}-N_{p}^{i} \phi_{j}^{p}\right) .
\end{aligned}
$$

From (1. 4) $)_{2}$ we have

$$
\text { (1. } 4)_{2}^{\prime} \quad N_{p}^{i} \phi_{j}^{p}=\xi^{i} N_{j}-2 t_{j i}^{i} \xi^{k} \text {. }
$$

On the other hand we have the following from (1. 1): 


$$
N_{p}^{i} \xi^{p} \eta_{j}=0
$$

Adding these two equations, we have

$$
N_{p}^{i}\left(\phi_{j}^{p}+\xi^{p} \eta_{j}\right)=\xi^{i} N_{j}-2 t_{j k}^{i} \xi^{k} .
$$

Multiplying $\left(\phi_{i l}^{j}-\xi^{j} \eta_{h}\right)$ on both sides, we have

$$
N_{h}^{i}=-\xi^{i} N_{p} \phi_{h}^{p}+2 t_{p q}^{i} \xi^{q} \phi_{h}^{p} .
$$

Since from (0.7) we can get

$$
\phi_{j}^{p} \phi_{k}^{q}\left(\eta_{q, p}-\eta_{p}, q\right)=\phi_{j}^{p} N_{p k}-\left(\eta_{j, k}-\eta_{k}, j\right)-N_{k} \eta_{j},
$$

by making use of which (0.4) can also be written as

$$
\begin{aligned}
t_{j k}^{i}=\frac{1}{4}\left\{-N_{j k}^{i}\right. & -4 \xi^{i}\left(\eta_{j, k}-\eta_{k}, j\right)+5 \xi^{i}\left(N_{j} \eta_{k}-N_{k} \eta_{j}\right) \\
& \left.-\xi^{i} N_{k} \eta_{j}+\xi^{i} \phi_{j}^{p} N_{p k}-N_{p}^{i}\left(\phi_{j}^{p} \eta_{k}-\phi_{k}^{p} \eta_{j}\right)\right\} .
\end{aligned}
$$

Contracting $\eta_{i}$ and then making use of $(1.3)_{2}$, we have

$$
\begin{aligned}
\eta_{i} t_{j k}^{i}=\frac{1}{4}\left\{-4\left(\eta_{j, k}\right.\right. & \left.-\eta_{k, j}\right)+4\left(N_{j} \eta_{k}-N_{k} \eta_{j}\right) \\
& \left.-\left(\eta_{i} N_{j k}^{i}+N_{k} \eta_{j}-\phi_{j}^{p} N_{p k}\right)\right\},
\end{aligned}
$$

from which and $(1.1)_{3}$ we have

$$
\eta_{p} t_{j k}^{p}=-\left(\eta_{j, k}-\eta_{k},{ }_{j}\right)+\left(N_{j} \eta_{k}-N_{k} \eta_{j}\right)=\eta_{i} t_{j k}^{i} .
$$

Substitute this formula to $(0.7)_{2}$, we have

$$
N_{j k}=N_{h} \phi_{k}^{h} \eta_{j}-N_{h} \phi_{j}^{h} \eta_{k}-\eta_{p} t_{h, j}^{p} \phi_{k}^{h}+\eta_{p} t_{h k}^{n} \phi_{j}^{h} .
$$

If we substitute (1.7) and (1. 4)' to (0.4) we have

(1. 9) $\quad P_{j k}^{i}=N_{j k}^{i}-\xi^{i}\left(N_{j} \eta_{k}-N_{k} \eta_{j}\right)$

$$
=\xi^{i} \phi_{j}^{p} \phi_{k}^{q} \eta_{l} t_{j q}^{l}+2 \eta_{k} t_{j p}^{i} \xi^{p}-2 \eta_{j} t_{k \mu}^{i} \xi^{p}+3 \xi^{i} \eta_{p} t_{j k}^{p}-4 t_{j k}^{i} .
$$

Finally, substitute (1.5) and (1.8) to $(0.6)_{2}$, we have

(1. 10) $Q_{j k}^{i}=-2 t_{\mu q}^{i} \xi^{q} \phi_{j}^{p} \eta_{k}+2 t_{p q}^{i} \xi^{q} \phi_{k}^{p} \eta_{j}-\xi^{i} \eta_{p} t_{n j}^{n} \phi_{k}^{h}+\xi^{i} \eta_{p} t_{h k}^{n} \phi_{j}^{n}$.

From (1. 7), (1. 9) and (1. 10) we see that the vanishing of $t_{i k}^{i}$ in (0.4) implies the vanishing of $t_{j k}^{i}, P_{j k}^{i}$ and $Q_{j k}^{i}$.

Conversely, suppose that $t_{j k}^{i}$ (or $\eta_{i} t_{j k}^{i}$ ) and $P_{j k}^{i}$ vanish simultaneously, then from (1. 7) and (1. 9) we have

$$
\eta_{p} t_{j k}^{p}=0
$$

and

$$
\eta_{k} t_{j p}^{i} \xi^{p}-\eta_{j} t_{k p}^{\prime} \xi^{p}-2 t_{j k}^{i}=0 .
$$

Multiply $\xi^{k}$ to this equation and contract with respect to $k$, we have 


$$
t_{j_{i}}^{i} \xi^{p}=0 .
$$

From the latter two equations we get $t_{j k}^{i}=0$. Thus we have

THEOREM 1.1. The vanishing of $t_{j k}^{i}$ in (0. 4) is equivalent to the simultaneous vanishing of the following two tensors:

$$
\left\{\begin{array}{l}
\left(N_{j} \eta_{k}-N_{k} \eta_{j}\right)-\left(\eta_{j, k}-\eta_{k}, j\right)=0, \\
N_{j k}^{i}-\xi^{i}\left(N_{j} \eta_{k}-N_{k} \eta_{j}\right)=0 .
\end{array}\right.
$$

Therefore these two relations are the necessary and sufficient condition for the

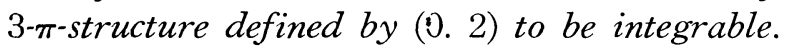

Now if $t_{j k}^{i}=0$ implies $N_{j_{i}}^{i}=0$ we have from above theorem $N_{j} \eta_{k}-N_{k} \eta_{j}$ $=0$, from which we have in turn $N_{j}=0$. Thus we have $\eta_{j, k}-\eta_{k},{ }_{j}=0$. Conversely, this implies $N_{j}=0$ and therefore $t_{j k}^{i}=0$ implies $N_{j k}^{t}=0$. By the same way it is evident that the necessary and sufficient condition for $N_{j k}^{i}=0$ to imply $t_{j k}^{i}=0$ is $\eta_{j, k}-\eta_{k},{ }_{j}=0$. Thus we have

THEOREM 1.2. In order that the vanishing of $N_{j k}^{i}$ is equivalent to the vanishing of $t_{j k}^{i}$, it is necessary and sufficient that $\eta_{j}$ is a gradient.

It is known [3] that $N_{j k}^{i}=0$ implies $N_{j}^{i}=0, \quad N_{j k}=0$ and $N_{j}=0$. So $N_{j k}^{i}=0$ also implies $P_{j k}^{i}=0$ and $Q_{j k}^{i}=0$ by virtue of $(0.6)$. But as is easily seen from (0.6), for $P_{j k}^{i}=0$ and $Q_{j k}^{i}=0$ to imply $N_{j k}^{i}=0$ it is necessary and sufficient that $N_{j}=0$. Thus we have

THEOREM 1.3. In order that the vanishing of $P_{j k}^{i}$ and $Q_{j k}^{i}$ is equivalent to the vanishing of $N_{j k}^{t}$ it is necessary and sufficient that $N_{j}=0$.

As the Lie derivative of $\eta_{j}$ is

$$
£(\xi) \eta_{j}=\xi^{k} \eta_{j, k}+\eta_{k} \xi_{, j}^{k}=\xi^{k}\left(\eta_{j, k}-\eta_{k},{ }_{j}\right)=N_{j}
$$

by virtue of (1. 1) $)_{1}$. From the meaning of Lie derivative and the Theorem 1.3 we have

THROREM 1.4. In a differentiable manifold having $(\phi, \xi, \eta)$-structure, for the vanishing of $N_{j k}^{i}$ it is necessary and sufficient that the following two conditions hold:

(i) the distribution defined by $\eta_{j}$ is invariant under the transformation generated by $\xi^{i}$,

(ii) the 2- $\pi$-structure defined by $(0.3)_{2}\left(\right.$ or $\left.(0.3)_{3}\right)$ is integrable.

Here the former $2-\pi$-structure is defined by two distributions, one of which is $(n+1)$-dimensional and consists of subspaces (of tangent spaces at each point) spanned by $\xi^{i}$ and proper vectors corresponding to proper value $i$ of $\phi_{j}^{i}$, and the other is $n$-dimensional and consists of subspaces spanned by proper vectors corresponding to proper value $-i$. 
2. If in the manifold having a $(\phi, \xi, \eta)$-structure there exists a positive definite Riemannian metric $g$ satisfying

$$
\left\{\begin{array}{l}
g_{i j} \xi^{i}=\eta_{j}, \\
g_{i j} \phi_{h}^{i} \phi_{k}^{j}=g_{h k}-\eta_{h} \eta_{k},
\end{array}\right.
$$

then the manifold is said to have a $(\phi, \xi, \eta, g)$-structure [1]. For any differentiable manifold $M^{2 n+1}$ with contact structure $\eta$, that is, a manifold over which a 1 form $\eta$ is defined and satisfying

$$
\eta \wedge(d \eta)^{n}=\eta \wedge d \eta \wedge \cdots \cdots \wedge d \eta \neq 0,
$$

one can find a $(\phi, \xi, \eta, g)$-structure such that $\eta_{i}$ is the one given as the coefficients of $\eta$ and

$$
d \eta=\phi=\frac{1}{2} \phi_{i j} d x^{i} \wedge d x^{j}, \phi_{i j}=\partial_{i} \eta_{j}-\partial_{j} \eta_{i} .
$$

From (2. 2) it follows that $\eta_{j}$ is not a gradient.

It is shown in [4] that if we consider a $(\phi, \xi, \eta, g)$-structure associated to the given contact structure $\eta$, then $N_{j}$ is identically zero. Thus we have from Theorem 1.1, Theorem 1.3 and Theorem 1.4 the following two corollaries:

COROLLARY 2.1. For a $(\phi, \xi, \eta, g)$-structure associated to a contact struct-

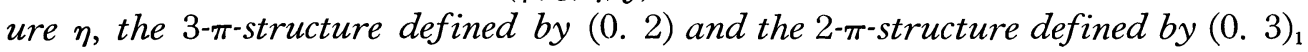
are both not integrable.

COROLLARY 2.2. For a differentiable manifold with $(\phi, \xi, \eta, g)$-structure associated with a contact structure $\eta$, the vanishing of $N_{j k}^{i}$ is characterized geometrically by the condition that the 2- $\pi$-structure defined by $(0.3)_{2}\left(\operatorname{or}(0.3)_{3}\right)$ is integrable.

3. Now we study on the existence of symmetric $(\phi, \xi, \eta)$-connection, which is by definition a symmetric connection leaving the tensor fields $\phi_{j}^{i}, \xi^{i}, \eta_{j}$ covariant constant [3]. Denote the covariant derivative with respect to a symmetric connection by; then the relations (0.7) hold good if we replace, in these relation by; and $\eta_{j, p}-\eta_{p, j}=\eta_{j ; p}-\eta_{p ; j}$. Thus if a differentiable manifold with $(\phi, \xi, \eta)$-structure has a symmetric $(\phi, \xi, \eta)$-connection, it follows that $N_{j k}^{i}, N_{j}^{i}$, $N_{j k}$ and $N_{j}$ all vanish, and $\eta_{j}$ is a gradient, so $t_{j k}^{i}$ also vanishes by virtue of (0.4). On the other hand, it is known [2] that if $N_{j}=0$, there is a $(\phi, \xi, \eta)$ connection whose torsion is equal to $t_{j k}^{i}$. Thus we have

THEOREM 3.1. Let $M^{2 n+1}$ be a manifold with $(\phi, \xi, \eta)$-structure. Then in order that there exists a symmetric $(\phi, \xi, \eta)$-connection it is necessary and sufficient that $\eta_{i}$ is a gradient and $t_{j k}^{i}=0$.

If we take account of Theorem 1.2, it is easily seen that Theorem 3.1 is equivalent to a Corollary in [3].

The following theorem is also closely related to the Theorem 3.1 , as $t_{j k}^{i}=0$ means the integrability of the $3-\pi$-structure which consists of a 1-dimensional distribution defined by $\xi^{i}$ and two $n$-dimensional distributions, one of 
which is defined by all subspaces spanned by the proper vectors at each point corresponding to proper value $i$ of $\phi_{j}^{i}$, and the other by those corresponding to proper value $-i$ [2] [5].

THEOREM 3.2. Let $M^{2 n+1}$ be a differentiable manifold with $(\phi, \xi, \eta)$-structure. If $M^{2 n+1}$ admits a symmetric $(\phi, \xi, \eta)$-connection, then (i) $\eta$ is a gradient, (ii) the induced almost complex structure on each integral submanifold of the distribution $\eta$ is invariant under the transformations generated by $\xi$ and (iii) each of these almost complex structure is integrable.

The closedness of $\eta$ and vanishing of $N_{j k}^{i}$ and $t_{j k}^{i}$ follow from the proof of Theorem 3.1, so we only require the proof of (ii). From the definition of Lie derivative, we have

$$
\begin{aligned}
& \mathcal{L}(\xi) \eta_{j}=\eta_{j ; k} \xi^{k}+\xi_{; j}^{h} \eta_{h}, \\
& \mathfrak{L}(\xi) \phi_{j}^{i}=\phi_{j ; k}^{i} \xi^{k}+\xi_{; ;}^{h} \phi_{h}^{i}-\xi_{; h}^{i} \phi_{j}^{h} .
\end{aligned}
$$

Therefore, from the assumption of the existence of symmetric $(\phi, \xi, \eta)$-connection we have

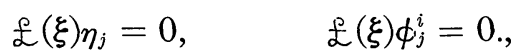

which means respectively that the integral submanifolds of the distribution $\eta$ are transformed by themselves and the structure $\phi$ is invariant under the transformations generated by $\xi$.

4. Finally we return to consider a differetiable manifold with $(\phi, \xi, \eta, g)$ structure. Denote the covariant derivative with respect to Christoffel's symbol constructed from $g_{i j}$ by $\nabla$. It is well known that the tensor

$$
\phi_{i j}=g_{i h} \phi_{j}^{h}
$$

is skew symmetric and the condition for the closedness of the exterior 2 -form

$$
\phi=\frac{1}{2} \phi_{i j} d x^{i} \wedge d x^{j}
$$

is as follows :

$$
\nabla_{k} \phi_{i j}+\nabla_{i} \phi_{j k}+\nabla_{j} \phi_{k i}=0
$$

Suppose the Riemann connection $\left\{\begin{array}{c}i \\ j k\end{array}\right\}$ is a $(\phi, \xi, \eta)$-connection, then $t_{j k}^{\prime}=0$ and both $\eta=\eta_{i} d x^{i}$ and $\phi$ are closed. The converse holds too. For from the assumption that $\eta$ is closed and $t_{j k}^{i}=0$ we have $N_{j k}^{i}=0$. From Theorem 3.1 and Theorem 3.2 it also follows that $\mathfrak{f}(\xi) \phi_{j}^{i}=0, \mathfrak{L}(\xi) \eta_{j}=0$. On the other hand it follows from the closedness of $\phi$ that $\mathfrak{L}(\xi) \phi_{j k}=0$. Applying Lie derivation with respect to $\xi^{i}$ to both sides of the relation $\phi_{j}^{i}=g^{i h} \phi_{h j}$ we have

$$
\left[£(\xi) g^{i h}\right] \phi_{h j}=0 .
$$


On the other hand we have

$$
\left[\mathfrak{L}(\xi) g^{i h}\right] \eta_{h} \eta_{j}=\left[\mathfrak{f}(\xi) \xi^{i}\right] \eta_{j}=0 .
$$

As $\phi_{i j}+\eta_{i} \eta_{j}$ is non-singular, from the latter two relations we have

$$
\mathfrak{L}(\xi) g^{i n}=0,
$$

which means that $\xi^{i}$ is a Killing vector field, that is,

$$
\nabla_{i} \eta_{j}+\nabla_{j} \eta_{i}=0 \text {. }
$$

This and the closedness of $\eta$ imply that

$$
\nabla_{i} \eta_{j}=0 \text {, }
$$

from which we have also the following

$$
\nabla_{i} \xi^{j}=\nabla_{i}\left(g^{j h} \eta_{h}\right)=0 .
$$

In the next place we shall prove that $\nabla_{k} \phi_{i j}=0$. From $N_{i j k}=g_{i h} N_{j k}^{\prime}=0$ and $\nabla_{j} \eta_{i}=0$ it follows that

$$
\phi_{k}^{h}\left(\nabla_{h} \phi_{i j}-\nabla_{j} \phi_{i h}\right)-\phi_{j}^{h}\left(\nabla_{h} \phi_{i k}-\nabla_{k} \phi_{i h}\right)=0 .
$$

Substitute from (4. 1) we have

$$
\phi_{k}^{h}\left(\nabla_{i} \phi_{h j}\right)-\phi_{j}^{h}\left(\nabla_{i} \phi_{h k}\right)=\nabla_{i}\left(\phi_{k}^{h} \phi_{h j}\right)-2 \phi_{j}^{h} \nabla_{i} \phi_{h k}=0 .
$$

But

$$
\nabla_{\imath}\left(\phi_{k}^{h} \phi_{h j}\right)=\nabla_{i}\left(g_{l h} \phi_{j}^{i} \phi_{k}^{h}\right)=\nabla_{i}\left(g_{j k}-\eta_{j} \eta_{k}\right)=0
$$

we have

$$
\left(\nabla_{i} \phi_{h k}\right) \phi_{j}^{h}=0 .
$$

On the other hand from $\phi_{h k} \xi^{h}=0$ and $\nabla_{i} \xi^{h}=0$ we have

$$
\left(\nabla_{i} \phi_{h k}\right) \xi^{h} \eta_{j}=0
$$

As $\phi_{j}^{h}+\xi^{h} \eta_{j}$ is non-singular, from these two relations we have

from which it follows

$$
\nabla_{i} \phi_{h k}=0
$$

$$
\nabla_{i} \phi_{k}^{i}=0
$$

Therefore we have

THEOREM 4.1. In order that the Riemannian connection of a $(\phi, \xi, \eta, g)$ structure to be $a(\phi, \xi, \eta)$-connection it is necessary and sufficient that the following three conditions hold:

(i ) $\eta$ is closed, (ii) $\phi$ is closed, (iii) $t_{j i}^{i}=0$. 
The following theorem is closely related to the above one:

THEOREM 4.2. Let $M^{2 n+1}$ be a differentiable manifold with $(\phi, \xi, \eta, g)$ structure. If the Riemannian connection is a $(\phi, \xi, \eta)$-connection, then (i) $\eta$ is closed, (ii) the induced almost Hermitian structure on each integral submanifold of the distribution $\eta$ is invariant under the transformations generated by $\xi$ and (iii) each of these almost Hermitian structure is Kählerian.

PROOF. From the Theorem 4.1 we have $t_{j k}^{i}=0$, so $t_{j k}^{i}=0$ holds too. Therefore the $2-\pi$-structture consisting the 1-dimensional distribution defined by $\xi^{i}$ and the $2 n$-dimensional one by $\eta_{j}$ is integrable, that is, for each point there is a neighborhood $U$, passing each point of which there is a unique 1-dimensional integral submanifold and a unique $2 n$-dimensional integral submanifold belonging to respective distribution. Moreover, in this neighborhood there is a local coordinate system $\left(x^{i}\right)(i=1, \cdots \cdots, 2 n+1)$ such that each 1-dimensional integral submanifold is expressed as $x^{a}=$ const. $(a, b, c=1, \cdots \cdots, 2 n)$ and each $2 n$-dimensional one as $x^{\Delta}=$ const. $(\Delta=2 n+1)$. In such local coordinate system, the projection tensor $\xi^{i} \eta_{j}$ to the direction of the 1-dimensional distribution has components $\xi^{i} \eta_{j}=0$ except for $\xi^{\Delta} \eta_{\Delta}=1$ because $\xi^{i} \eta_{i}=1$. Therefore, we have the following components of tensor (vector) fields in this coordinates system :

$$
\begin{array}{cc}
\xi^{i}=\delta_{\Delta}^{\prime}, & \eta_{j}=\delta_{i}^{\Delta}, \\
\left(\phi_{j}^{i}\right)=\left(\begin{array}{cc}
\phi_{b}^{n} & 0 \\
& \\
0 & 0
\end{array}\right), \quad\left(g_{i j}\right)=\left(\begin{array}{cc}
g_{a b} & 0 \\
0 & 0
\end{array}\right) .
\end{array}
$$

Then $\phi_{b}^{n}\left(x^{c}, x^{\lrcorner}\right)$and $g_{a b}\left(x^{c}, x^{\lrcorner}\right)$are the almost complex structure and the almost Hermitian metric on integral submanifolds in consideration.

From the assumptions

$$
\nabla_{k} \xi^{i}=0, \quad \nabla_{k} \eta_{j}=0,
$$

we can easily get

$$
\left\{\begin{array}{c}
i \\
\Delta k
\end{array}\right\}=0, \quad\left\{\begin{array}{c}
\Delta \\
j k
\end{array}\right\}=0
$$

and from the assumptions

$$
\nabla_{k} \phi_{j}^{i}=0, \quad \nabla_{k} g_{i j}=0,
$$

we know that $\phi_{j}^{i}$ and $g_{i j}$ do not depend upon $x^{\Delta}$. This shows that both $\phi$ and $g$ are invariant under the transformations generated by $\xi^{i}$. And secondly we see that

$$
\frac{\partial \phi_{b}^{n}}{\partial x^{c}}+\left\{\begin{array}{c}
a \\
e c
\end{array}\right\} \phi_{b}^{e}-\left\{\begin{array}{c}
e \\
b c
\end{array}\right\} \phi_{e}^{a}=0
$$


which shows that the induced almost Hermitian structure is Kählerian.

\section{REFERENCES}

[1] S. SASAKI, On differentiable manifolds with certain structures which are closely related to almost contact structure I. Tôhoku Math. Jour., 12(1960).

[2] C. J. Hsu, Note on $(\phi, \xi, \eta)$-structure. Tôhoku Math. Jour., 13(1961).

[3] S. SASAKI AND Y. HATAKEYAMA, On differentiable manifolds with certain structures which are closely related to almost contact structure II. Tòhoku Math. Jour., 13(1961).

[4] S. SASAKI AND Y. HATAKEYAMA, On differentiable manifolds with contact metric structures. To appear in Jour. Japan Math. Soc.

[5] C. J. Hsu, On some properties of $\pi$-structures of differentiable manifolds. Tôhoku Math. Jour., $12(1960)$.

TÔHOKU UNIVERSITY AND NATIONAL TAIWAN UNIVERSITY

NATIONAL TAIWAN UNIVERSITY. 